\title{
Vacancy and interstitial depth profiles in ion-implanted silicon
}

\author{
P. Lévêque, H. Kortegaard Nielsen, P. Pellegrino, and A. Hallén \\ Solid State Electronics, Royal Institute of Technology, Electrum 229, SE-16440 Kista-Stockholm, Sweden \\ B. G. Svensson a) and A. Yu. Kuznetsov \\ Physical Electronics, Department of Physics, Oslo University, P.B. 1048 Blindern, N-0316 Oslo, Norway \\ and Solid State Electronics, Royal Institute of Technology, Electrum 229, SE-16440 Kista- \\ Stockholm, Sweden \\ J. Wong-Leung and C. Jagadish \\ Department of Electronic Materials Engineering, Research School of Physical Sciences and Engineering, \\ Australian National University, Canberra ACT 0200, Australia \\ V. Privitera \\ CNR-IMM, Stradale Primosole 50, 95121 Catania, Italy
}

(Received 12 February 2002; accepted 17 October 2002)

\begin{abstract}
An experimental method of studying shifts between concentration-versus-depth profiles of vacancyand interstitial-type defects in ion-implanted silicon is demonstrated. The concept is based on deep level transient spectroscopy measurements utilizing the filling pulse variation technique. The vacancy profile, represented by the vacancy-oxygen center, and the interstitial profile, represented by the interstitial carbon-substitutional carbon pair, are obtained at the same sample temperature by varying the duration of the filling pulse. The effect of the capture in the Debye tail has been extensively studied and taken into account. Thus, the two profiles can be recorded with a high relative depth resolution. Using low doses, point defects have been introduced in lightly doped float zone $n$-type silicon by implantation with $6.8 \mathrm{MeV}$ boron ions and $680 \mathrm{keV}$ and $1.3 \mathrm{MeV}$ protons at room temperature. The effect of the angle of ion incidence has also been investigated. For all implantation conditions the peak of the interstitial profile is displaced towards larger depths compared to that of the vacancy profile. The amplitude of this displacement increases as the width of the initial point defect distribution increases. This behavior is explained by a simple model where the preferential forward momentum of recoiling silicon atoms and the highly efficient direct recombination of primary point defects are taken into account. (C) 2003 American Institute of Physics. [DOI: 10.1063/1.1528304]
\end{abstract}

\section{INTRODUCTION}

Ion implantation has been used as a doping technique in silicon device processing for more than 30 years $^{1}$ and continues to be a standard "front end" process in the early 21 st century. However, with increasing miniaturization of silicon integrated circuits, the demands on well-controlled dopant distributions (in three dimensions) become extremely severe and ion implantation may not remain the prime doping technique because of the inherent limitations associated with defect generation, postimplant annealing and dopant diffusion. On the other hand, few viable alternatives exist and, consequently, this has stimulated a substantial activity to improve the ability in controlling implanted dopant distributions. It has been established that anomalous diffusion of $\mathrm{B}$ and $\mathrm{P}$ during postimplant annealing is due to the excess of point defects generated by the implantation. ${ }^{2-7}$ Hence, knowledge about the initial distributions of excess point defects is crucial in order to understand and model the enhanced dopant diffusion.

Because of a preferential momentum transfer from the impinging ions to the silicon atoms in the forward direction,

${ }^{\text {a)} E l e c t r o n i c ~ m a i l: ~ b . g . s v e n s s o n @ f y s . u i o . n o ~}$ a slight displacement between the vacancy $(V)$ and Si selfinterstitial (I) distributions is predicted. ${ }^{8}$ Some attempts have been made to observe this shift experimentally by comparing results from spreading resistance measurements on $n$ - and $p$-type samples. ${ }^{9}$ A different approach was recently introduced for direct comparison of the depth profiles of specific vacancy- and interstitial-type defects. ${ }^{10}$ The same concept has been used and further developed in this work. We have employed deep level transient spectroscopy (DLTS) (Ref. 11) to study the shift between the concentration versus depth profiles of the vacancy-oxygen center $(\mathrm{VO})$ and the interstitial carbon-substitutional carbon pair $\left(\mathrm{C}_{i} \mathrm{C}_{s}\right)$ in $n$-type samples implanted with $\mathrm{MeV}$ protons or boron ions. $\mathrm{VO}$ and $\mathrm{C}_{i} \mathrm{C}_{s}$ both give rise to an acceptor level at $\sim 0.17 \mathrm{eV}$ below the conduction-band edge $\left(E_{c}\right)$, but they can readily be resolved by variation of the filling pulse duration $\left(\tau_{p}\right)$. However, variation of $\tau_{p}$ influences the rate of charge carrier capture in the so-called Debye tail and an effect of $\tau_{p}$ occurs on the width of the probed region for a given pulse amplitude at a fixed bias voltage. Hence, we have made substantial experimental efforts to ensure that large enough $\tau_{p}$ values are used to saturate the charge carrier trapping in the Debye tail but short enough to separate the $\mathrm{VO}$ and $\mathrm{C}_{i} \mathrm{C}_{s}$ contributions. For all implantation conditions, the $I$ depth distribution 
TABLE I. Survey of the implantation conditions used in this study.

\begin{tabular}{lccccc}
\hline \hline Sample identification & Ion & Energy & $\alpha$ & Dose $\left(/ \mathrm{cm}^{2}\right)$ & Dose rate $\left(/ \mathrm{cm}^{2} \mathrm{~s}\right)$ \\
\hline $\mathrm{B}(6.8 \mathrm{MeV}) 7 d$ & $\mathrm{~B}$ & $6.8 \mathrm{MeV}$ & $7^{\circ}$ & $1.4 \times 10^{8}$ & $1 \times 10^{8}$ \\
$\mathrm{~B}(6.8 \mathrm{MeV}) 40 d$ & $\mathrm{~B}$ & $6.8 \mathrm{MeV}$ & $40^{\circ}$ & $1 \times 10^{8}$ & $1 \times 10^{8}$ \\
$\mathrm{H}(680 \mathrm{keV}) 7 d$ & $\mathrm{H}$ & $680 \mathrm{keV}$ & $7^{\circ}$ & $3 \times 10^{9}$ & $1 \times 10^{8}$ \\
$\mathrm{H}(680 \mathrm{keV}) 40 d$ & $\mathrm{H}$ & $680 \mathrm{keV}$ & $40^{\circ}$ & $4 \times 10^{9}$ & $1 \times 10^{8}$ \\
$\mathrm{H}(1.3 \mathrm{MeV}) 7 d$ & $\mathrm{H}$ & $1.3 \mathrm{MeV}$ & $7^{\circ}$ & $1 \times 10^{10}$ & $1 \times 10^{9}$ \\
\hline \hline
\end{tabular}

monitored by $\mathrm{C}_{i} \mathrm{C}_{s}$ is deeper than the $V$ one monitored by VO. Moreover, the observed shift between the $I$ and $V$ depth distributions increases as the primary defect distribution width increases. This observation is explained by a simple model where the preferential forward momentum of recoiling silicon atoms and the highly efficient direct recombination of primary point defects are taken into account.

\section{EXPERIMENTAL PROCEDURE}

The samples used in this study were cut from the same high-purity $n$-type $(75 \Omega \mathrm{cm}$ ) float zone (FZ) silicon wafer. The main impurities, oxygen and carbon, have an estimated concentration of $\sim 5 \times 10^{15} \mathrm{~cm}^{-3}$ according to Fourier transform infrared absorption measurements.

The samples were implanted at room temperature with boron or hydrogen ions using the implantation parameters given in Table I. The hydrogen $(1.3 \mathrm{MeV})$ implantation was undertaken at the Svedberg Laboratory in Uppsala. A 12$\mu \mathrm{m}$-thick aluminum foil was placed immediately in front of the samples in order to obtain a mean projected range of $\sim 12$ $\mu \mathrm{m}$ in the silicon crystal, according to transport of ions in matter (TRIM 98) calculations, ${ }^{12}$ suitable to monitor the defect distributions by DLTS. The other samples were implanted at the Australian National University in Canberra with implantation energies such that the mean projected range was smaller than or equal to $12 \mu \mathrm{m}$. Both accelerators are of tandem type and equipped with an $X-Y$ scanning facility for homogeneous area coverage. ${ }^{13}$ The ion doses were tuned to avoid doping compensation effects. The error in the nominal dose did not exceed $15 \%$ for the hydrogen implants but was as high as $50 \%$ for the boron implants because of the short implantation times required. In addition to the ion type and energy, the angle of incidence $(\alpha)$ between the ion beam and the surface normal was also varied from a standard $7^{\circ}$ tilt to a $40^{\circ}$ tilt.

After implantation, the samples were chemically cleaned using a standard procedure including a final dip in diluted hydrofluoridric acid. Schottky barrier (SB) junctions were subsequently grown at low temperature $\left(<40^{\circ} \mathrm{C}\right)$ by thermal evaporation of gold through a metal mask at a base pressure $<2 \times 10^{-6}$ Torr.

DLTS analysis was performed using a refined version of a setup described elsewhere. ${ }^{14}$ Six DLTS spectra with a window rate ranging from $(100 \mathrm{~ms})^{-1}$ to $(3200 \mathrm{~ms})^{-1}$ were simultaneously recorded during one single temperature scan from 77 to $300 \mathrm{~K}$ yielding an overview of the electrically active defects present in the upper half of the band gap. The steady-state reverse bias and filling pulse voltages were chosen such that the whole defect profiles were covered and the filling pulse duration was set to $50 \mathrm{~ms}$ in order to fully saturate traps with a capture cross section as low as $10^{-18}-10^{-19} \mathrm{~cm}^{2}$.

Filling pulse and concentration versus depth measurements were performed by selecting one of the six rate windows and holding the temperature constant within $\pm 0.5 \mathrm{~K}$ at the maximum of the DLTS peak of interest. In the former measurements, the amplitude of the DLTS signal was then measured as a function of the filling pulse duration ranging from $5 \times 10^{-8}$ to $4 \mathrm{~s}$. In the latter measurements, the steadystate reverse bias voltage was kept constant while gradually increasing the majority carrier pulse amplitude. The depth profile was then extracted from the dependence of the DLTS signal on the pulse amplitude, ${ }^{11}$ where the voltages used were converted into depth by the conventional square-root dependence for a SB junction, and in particular, the effect of charge carrier filling in the Debye tail was studied in detail. Further, the compensation of dopants was investigated for each sample and found to be negligible, especially at $79 \mathrm{~K}$ where the concentration versus depth profiles were recorded.

\section{RESULTS AND DISCUSSION}

\section{A. $E_{c}-0.17 \mathrm{eV}$ level}

A typical DLTS spectrum for majority-carrier traps introduced in $n$-type FZ silicon samples by boron implantation is shown in Fig. 1. Three major levels appear at $\sim 0.42$, $\sim 0.23$, and $\sim 0.17 \mathrm{eV}$ below the conduction-band edge $\left(E_{c}\right)$. The two former ones are primarily ascribed to the single and double negative charge states of the divacancy $\left(V_{2}\right)$ center, respectively. ${ }^{15-17}$ The $E_{c}-0.42 \mathrm{eV}$ level also contains some

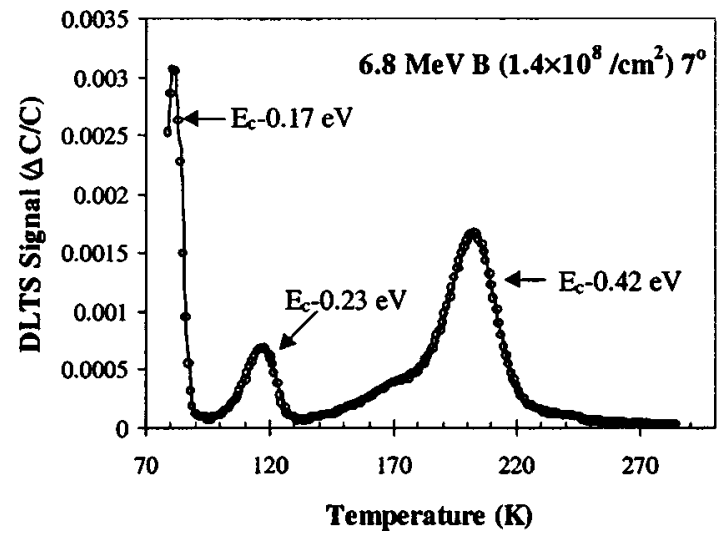

FIG. 1. DLTS spectrum of an $n$-type FZ sample implanted with 1.4 $\times 10^{8} / \mathrm{cm}^{2} \mathrm{~B}(6.8 \mathrm{MeV})$ ions at an angle of incidence of $7^{\circ}$ relative to the surface normal. The rate window is $(200 \mathrm{~ms})^{-1}$ and the filling pulse duration is $50 \mathrm{~ms}$. 


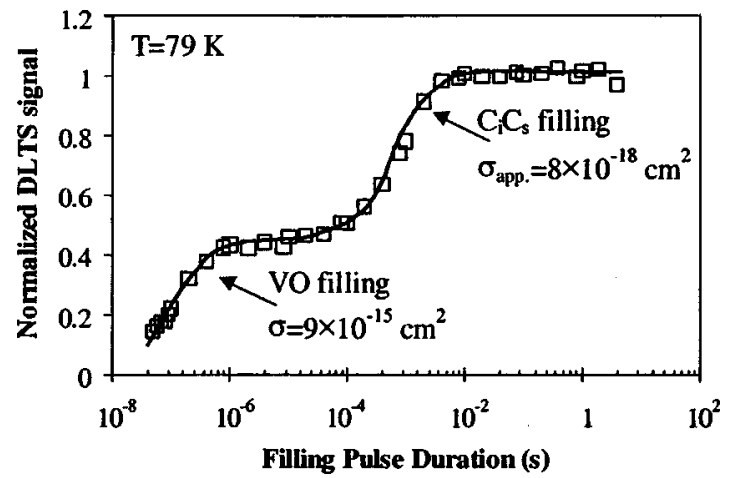

FIG. 2. Amplitude of the $E_{c}-0.17 \mathrm{eV}$ level as a function of the filling pulse duration $\left(\tau_{p}\right)$ normalized to the value at $\tau_{p}=1 \mathrm{~s}$. The sample temperature during measurements was $79 \mathrm{~K}$ and the sample is the same one as in Fig. 1. The rate window is $(400 \mathrm{~ms})^{-1}$.

contribution from the vacancy-phosphorous defect (VP) as shown by subsequent annealing. In the following, we will focus on the $E_{c}-0.17 \mathrm{eV}$ level. Two contributions to this level can be easily resolved in samples where the concentrations of oxygen and carbon are similar. The amplitude of the $E_{c}-0.17 \mathrm{eV}$ level in a boron implanted $\left(\alpha=7^{\circ}\right)$ sample as a function of $\tau_{p}$ is given in Fig. 2 with the amplitude values normalized to that obtained at $1 \mathrm{~s}$. The amplitude increases continuously with $\tau_{p}$ in the range from $5 \times 10^{-8}$ to $10^{-6} \mathrm{~s}$ and then saturates until $\tau_{p}$ reaches $10^{-4} \mathrm{~s}$. For $\tau_{p}>10^{-4} \mathrm{~s}$, it increases again until a second plateau is reached around $\tau_{p} \approx 3 \times 10^{-3} \mathrm{~s}$. The first part $\left(5 \times 10^{-8}<\tau_{p}<10^{-4} \mathrm{~s}\right)$ corresponds to the filling of the well-known VO center ${ }^{18}$ with a large capture cross section $\left(\sim 9 \times 10^{-15} \mathrm{~cm}^{2}\right)$ while the second part $\left(\tau_{p}>10^{-4} \mathrm{~s}\right)$ is related to the bistable $\mathrm{C}_{i} \mathrm{C}_{s}$ pair $^{19}$ with a small apparent capture cross section $(\sim 8$ $\times 10^{-18} \mathrm{~cm}^{2}$ ). The configurational-coordinate energy diagram for the acceptor state of the $\mathrm{C}_{i} \mathrm{C}_{s}$ pair, as determined by Song et al. ${ }^{19}$ is shown in Fig. 3. The $\mathrm{C}_{i} \mathrm{C}_{s}$ pair can exist in two different configurations labeled $\mathrm{A}$ and $\mathrm{B}$. Cooling the sample under reverse bias conditions, the most energetically favorable configuration is $\mathrm{B}^{0}$ giving rise to an acceptor level around $0.11 \mathrm{eV}$ below $E_{c}$, which is too shallow to be monitored by our setup. On the other hand, when $\tau_{p}$ is long enough, the $\mathrm{C}_{i} \mathrm{C}_{s}$ pair can switch from the $\mathrm{B}^{-}$to the $\mathrm{A}^{-}$ configuration, which is the most favorable one for $\mathrm{C}_{i} \mathrm{C}_{s}{ }^{-}$.

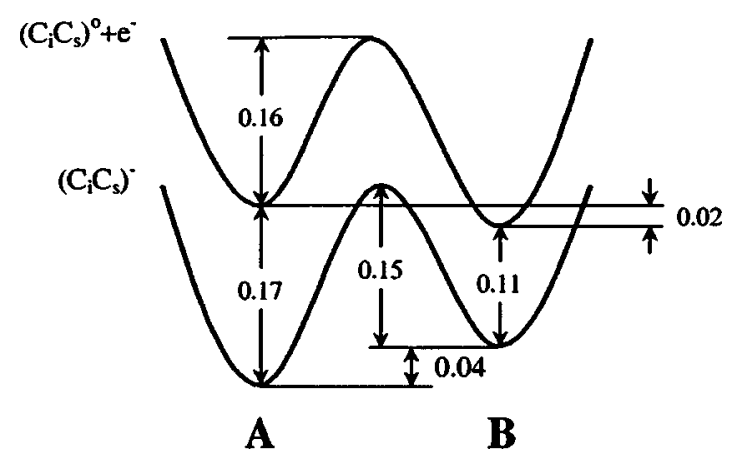

FIG. 3. Configurational-coordinate energy diagram for the acceptor state of the $\mathrm{C}_{i} \mathrm{C}_{s}$ pair as proposed by Song et al. (see Ref. 19).
The transition $\mathrm{B}^{-} \rightarrow \mathrm{A}^{-}$is associated with the critical time $\tau_{c}$ determined by Jellison ${ }^{20}$ and can be expressed by

$$
\tau_{c}=7.3 \times 10^{-13} \exp [(0.145 \mathrm{eV}) / k T](\mathrm{s}),
$$

where $k$ and $T$ are the Boltzmann constant and the absolute temperature, respectively. In Fig. 2, $T$ is equal to $79 \mathrm{~K}$ leading to $\tau_{c}=1.3 \times 10^{-3} \mathrm{~s}$. When $\tau_{p}$ is longer than this value, $\mathrm{B}^{-}$transforms to $\mathrm{A}^{-}$and the electron emission from $\mathrm{C}_{i} \mathrm{C}_{s}{ }^{-}$ can be monitored at $79 \mathrm{~K}$, giving rise to a level overlapping with VO but with a much smaller apparent capture cross section. In practice, by changing the filling pulse duration, we are able to probe $\mathrm{VO}$ alone $\left(5 \times 10^{-8}<\tau_{p}<10^{-4} \mathrm{~s}\right)$ or the total contribution of $\mathrm{VO}$ and $\mathrm{C}_{i} \mathrm{C}_{s}\left(\tau_{p}>10^{-4} \mathrm{~s}\right)$, keeping all the other experimental parameters unchanged. Here, it should be emphasized that both the $\mathrm{VO}$ and $\mathrm{C}_{i} \mathrm{C}_{s}$ contributions are due to acceptor-like levels, and therefore, no Poole-Frenkel effect is expected for the two levels. ${ }^{21,22}$

$\mathrm{VO}$ is formed through the capture of migrating monovacancies $(V)$ by interstitial oxygen atoms $\left(\mathrm{O}_{i}\right)$. With an interstitial oxygen concentration $\left(\left[\mathrm{O}_{i}\right]\right)$ in the range of 5 $\times 10^{15} \mathrm{~cm}^{-3}$, the average migration length of $V$ before trapping by $\mathrm{O}_{i}$ is of the order of $0.1 \mu \mathrm{m}$. Since $\left[\mathrm{O}_{i}\right] \gg[\mathrm{VO}]$ for the low dose regime studied, the production of $\mathrm{VO}$ reflects the amount of vacancies escaping direct annihilation. Therefore, $\mathrm{VO}$ can be considered as a primary defect and as a monitor of the formation of vacancies. The $\mathrm{C}_{i} \mathrm{C}_{s}$ pair is formed by the trapping of a migrating interstitial carbon $\left(\mathrm{C}_{i}\right)$ by a substitutional carbon $\left(\mathrm{C}_{s}\right) . \mathrm{C}_{i}$ 's are generated by the interaction of implantation-induced self-interstitials $(I)$ and the $\mathrm{C}_{s}$ atoms and can be regarded as a monitor of the free self-interstitials not recombining with vacancies. $\mathrm{C}_{i}$ is less mobile than $I$ and $V$ but exhibits an appreciable mobility at room temperature with a diffusion coefficient of $10^{-15} \mathrm{~cm}^{2} / \mathrm{s}$ at $300 \mathrm{~K} .{ }^{23}$ With $\left[\mathrm{C}_{s}\right] \sim 5 \times 10^{15} \mathrm{~cm}^{-3}$, the $\mathrm{C}_{i} \mathrm{C}_{s}$ pairs are formed within a few days after implantation and the effective diffusion length of $\mathrm{C}_{i}$ is on the order of $0.1 \mu \mathrm{m}$. The $\mathrm{C}_{i} \mathrm{C}_{s}$ pair is predominantly observed in oxygen lean samples where the competing $\mathrm{O}_{i}$ trap for migrating $\mathrm{C}_{i}$ is less effective. The $\mathrm{C}_{i} \mathrm{C}_{s}$ pair is one of the few well-characterized defects of interstitial type with an energy level in the upper half of the band gap.

As indicated in Fig. 2, $\left[\mathrm{C}_{i} \mathrm{C}_{s}\right]$ and [VO] are similar in the boron-implanted samples and the same holds for the hydrogen-implanted samples. This condition is necessary in order to monitor accurately the depth profiles of both $\mathrm{C}_{i} \mathrm{C}_{s}$ and VO. Moreover, for depth profiling where filling pulses with different durations are employed, one has to consider charge carrier trapping in the Debye tail, as outlined in the following section.

\section{B. Capture in the Debye tail}

The DLTS technique is basically a "pulsed-bias" technique where the time evolution $\Delta C$ of the capacitance of a reverse biased diode is recorded after the application of a filling pulse with duration $\tau_{p}$. This pulse shrinks the spacecharge region of the diode and fills the deep traps in the 


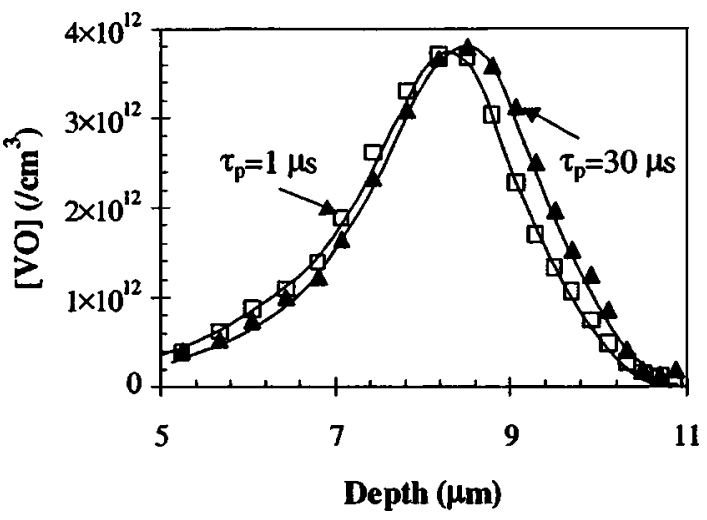

FIG. 4. Concentration vs depth profiles for the VO center obtained with a filling pulse duration $\left(\tau_{p}\right)$ of $1 \mu$ s (open squares) and $30 \mu$ s (black triangles), respectively. The sample used is the same one as in Fig. 1.

neutralized volume. If $\Delta C$ is only due to the filling of a single deep trap, a purely exponential filling kinetics is expected.

$$
\Delta C=\Delta C_{\max }\left[1-\exp \left(-\tau_{p} / \tau_{t}\right)\right]
$$

where $\Delta C_{\max }$ is the maximum change in capacitance for complete filling and $\tau_{t}$ the capture time constant of the deep trap. Equation (2) is, however, a simplification since experimentally two components are normally always observed: a fast one [purely exponential, as expected from Eq. (2)] and a slow one that corresponds to capture in the remaining potential barrier during the pulse. ${ }^{24}$ The slow part is due to the distribution of free carriers near the edge of the space-charge region, i.e., the so-called Debye tail. An analytical expression for the filling in the Debye tail has been put forward in the case of a homogeneous distribution of deep traps ${ }^{25}$ but not for an inhomogeneous distribution (like in ion-implanted samples). The aim of the present article is not to determine the capture kinetics in the Debye tail for an inhomogeneous trap distribution but this effect occurs in our measurements and has to be considered. Figure 4 represents the concentration versus depth profile of the $E_{c}-0.17 \mathrm{eV}$ level for two different filling pulse durations of 1 and $30 \mu$ s, respectively, in the boron-implanted sample $\left(\alpha=7^{\circ}\right.$, the same sample as in Fig. 2). As expected according to Fig. 2, the profile contains only the VO contribution and the maximum VO concentration is unchanged when $\tau_{p}$ increases from 1 to $30 \mu$ s. On the other hand, the position of the peak changes because of the variation of the filling in the Debye tail as a function of $\tau_{p}$, and in fact, this holds for both $\mathrm{VO}$ and $\mathrm{C}_{i} \mathrm{C}_{s}$, as shown in the following section.

\section{Determination of the true concentration versus depth profiles}

Depending on the filling pulse duration used for the concentration versus depth profiling of the $E_{c}-0.17 \mathrm{eV}$ level, we can measure the VO concentration and a certain percentage of the $\mathrm{C}_{i} \mathrm{C}_{s}$ concentration ranging from $0 \%$ to $100 \%$. In order to resolve accurately any shift between the two profiles, the extracted depth of the total $E_{c}-0.17 \mathrm{eV}$ peak maximum $\left(d_{\max }\right)$ is determined as a function of $\tau_{p}$, as shown in

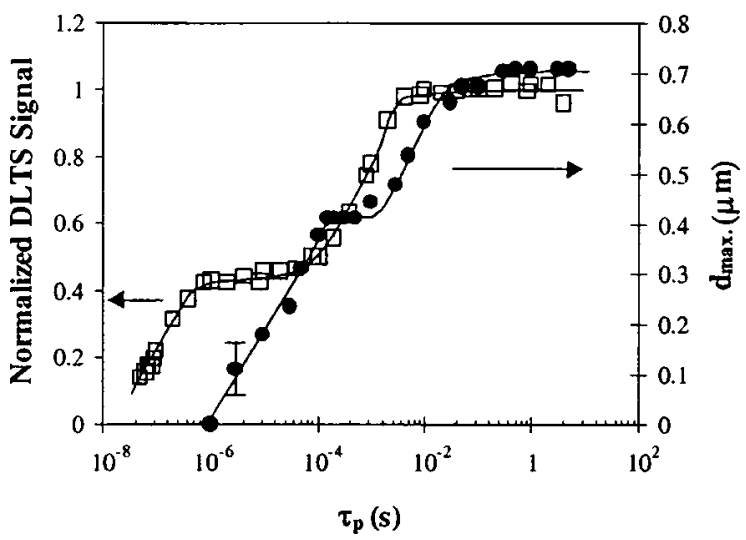

FIG. 5. Position $\left(d_{\max }\right)$ of the maximum concentration of the $E_{c}-0.17 \mathrm{eV}$ depth profile (black circles) and the normalized DLTS amplitude of the same level (open squares) as a function of the filling pulse duration $\left(\tau_{p}\right)$. The sample used is the same one as in Fig. 1.

Fig. 5 for the boron $\left(\alpha=7^{\circ}\right)$ implanted sample. The value of $d_{\max }$ for the lowest $\tau_{p}$ employed ( $\left.1 \mu \mathrm{s}\right)$ is used as a reference value and put equal to zero. Further, the amplitude of the $E_{c}-0.17 \mathrm{eV}$ level as a function of $\tau_{p}$ is also included in Fig. 5. An accuracy of $\pm 0.05 \mu \mathrm{m}$ is deduced for $d_{\max }$ from different measurements on the same sample.

For small values of $\tau_{p}\left(\tau_{p} \leqslant 10^{-4} \mathrm{~s}\right)$, the $E_{c}-0.17 \mathrm{eV}$ level contains only the VO contribution and $d_{\max }$ increases continuously. This increase is due to increasing filling of $\mathrm{VO}$ in the Debye tail, as discussed in Sec. III B. For $\tau_{p}$ ranging from $10^{-4}$ to $10^{-3}$, i.e., for a $\mathrm{C}_{i} \mathrm{C}_{s}$ contribution ranging from $0 \%$ to $60 \%$ of its maximum concentration, $d_{\max }$ remains constant. This behavior indicates clearly that the filling of VO in the Debye tail is completed when $\tau_{p} \geqslant 10^{-4} \mathrm{~s}$ or in other words that the extracted profile for the $E_{c}-0.17 \mathrm{eV}$ level when $\tau_{p}=(1-2) \times 10^{-4} \mathrm{~s}$ can be considered as the reference (true) VO profile. This conclusion is further supported by the calculations performed in Sec. III D. For $\tau_{p} \geqslant 10^{-3} \mathrm{~s}, d_{\max }$ increases again because of the filling of the $\mathrm{C}_{i} \mathrm{C}_{s}$ pair in the Debye tail. Indeed, the $\mathrm{VO}$ capture cross section $[\sigma(\mathrm{VO})$ $\left.\sim 9 \times 10^{-15} \mathrm{~cm}^{2}\right]$ and the $\mathrm{C}_{i} \mathrm{C}_{s}$ apparent capture cross section $\left[\sigma_{\text {app }}\left(\mathrm{C}_{i} \mathrm{C}_{s}\right) \sim 8 \times 10^{-18} \mathrm{~cm}^{2}\right]$ are very different and, consequently, the increase in $d_{\max }$ due to the filling in the Debye tail takes place in different $\tau_{p}$ intervals for the two types of defects. For $\tau_{p} \geqslant 10^{-1} \mathrm{~s}, d_{\max }$ saturates again, indicating that the filling in the Debye tail is completed for the $\mathrm{C}_{i} \mathrm{C}_{s}$ pair.

The profile of the $E_{c}-0.17 \mathrm{eV}$ level for $\tau_{p}=1 \mathrm{~s}$ was chosen as the reference profile for the total $\mathrm{VO}+\mathrm{C}_{i} \mathrm{C}_{s}$ contributions. The $\mathrm{C}_{i} \mathrm{C}_{s}$ pair profile itself is deduced by subtraction of the $E_{c}-0.17 \mathrm{eV}$ level profile measured at $\tau_{p}=1 \mathrm{~s}$ $\left(\mathrm{VO}+\mathrm{C}_{i} \mathrm{C}_{s}\right)$ by that at $\tau_{p}=2 \times 10^{-4} \mathrm{~s}(\mathrm{VO})$. It is then possible to determine the shift $(\Delta)$ that may exist between the $\mathrm{VO}$ and the $\mathrm{C}_{i} \mathrm{C}_{s}$ pair distributions. An example of such a measurement for the boron $\left(\alpha=7^{\circ}\right)$ implanted sample is shown in Fig. 6. The same procedure has been used for all the implanted samples leading to the results displayed in Table II.

A shift is observed between the $\mathrm{VO}$ and $\mathrm{C}_{i} \mathrm{C}_{s}$ distributions for all the samples used in this study and the $\mathrm{C}_{i} \mathrm{C}_{s}$ 


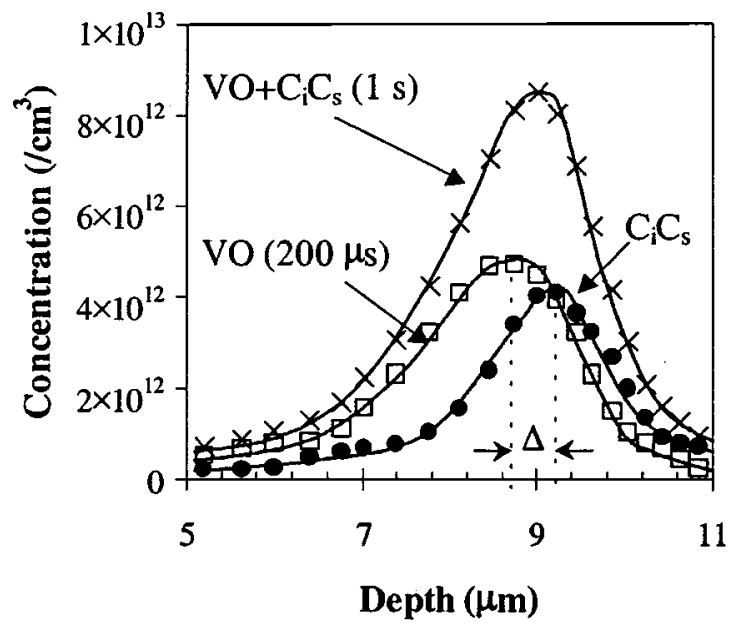

FIG. 6. Determination of the true shift $(\Delta)$ between the $\mathrm{C}_{i} \mathrm{C}_{s}$ (black circles) and the $\mathrm{VO}$ (open squares) depth profiles from the total $\mathrm{VO}+\mathrm{C}_{i} \mathrm{C}_{s}$ (crosses) depth profile. The sample used is the same as in Fig. 1 at a temperature of 79 $\mathrm{K}$ and a rate window of $(400 \mathrm{~ms})^{-1}$. The filling pulse durations used $\left(\tau_{p}\right)$ are indicated in the figure.

distribution, which reflects the $I$ distribution, is always the deepest one. $\Delta$ is substantially larger than the diffusion lengths involved for $V, I$, and $\mathrm{C}_{i}$ at room temperature and is predominantly ascribed to the preferential momentum transfer in the forward direction to the recoiling silicon atoms. ${ }^{10}$ It is quite surprising that, according to this interpretation, such a "light" implantation as $680 \mathrm{keV}$ protons with $\alpha=40^{\circ}$ leads to a substantial shift. Another surprising result is that for implants using the same type of ion and energy, an increase of $\alpha$ gives a larger $\Delta$. To gain further insight and understanding of these results, simple calculations have been performed in order to model the behavior of $d_{\max }$ vs $\tau_{p}$ for different values of $\Delta$.

\section{Calculation of the depth maximum}

The starting assumptions are (i) the $\mathrm{VO}$ and $\mathrm{C}_{i} \mathrm{C}_{s}$ distributions have the same shape as a function of depth and the same maximum concentration and (ii) $\mathrm{VO}$ and $\mathrm{C}_{i} \mathrm{C}_{s}$ exhibit the same capture kinetics in the Debye tail but in different $\tau_{p}$ intervals because of the difference between $\sigma(\mathrm{VO})$ and $\sigma_{\text {app }}\left(\mathrm{C}_{i} \mathrm{C}_{s}\right)$. The first assumption is supported by Fig. 6 while the effect of the filling in the Debye tail [in the following, called the Debye Tail Shift (DTS)] is taken into account according to Fig. 7; for both DTS(VO) and DTS $\left(\mathrm{C}_{i} \mathrm{C}_{s}\right)$ the dependence as a function of $\tau_{p}$ is based on the results in Fig. 5. Here, it should be emphasized that $\sigma_{\text {app }}\left(\mathrm{C}_{i} \mathrm{C}_{s}\right)$ does not reflect the rate of electron capture for $\mathrm{C}_{i} \mathrm{C}_{s}$ but rather the rate

TABLE II. Measured shift $(\Delta)$ between the $C_{i} C_{s}$ and VO concentration vs depth profiles.

\begin{tabular}{lc}
\hline \hline Sample identification & $\Delta(\mu \mathrm{m})( \pm 0.05 \mu \mathrm{m})$ \\
\hline $\mathrm{B}(6.8 \mathrm{MeV}) 7 d$ & 0.45 \\
$\mathrm{~B}(6.8 \mathrm{MeV}) 40 d$ & 0.57 \\
$\mathrm{H}(680 \mathrm{keV}) 7 d$ & 0.50 \\
$\mathrm{H}(680 \mathrm{keV}) 40 d$ & 0.60 \\
$\mathrm{H}(1.3 \mathrm{MeV}) 7 d$ & 0.90 \\
\hline \hline
\end{tabular}

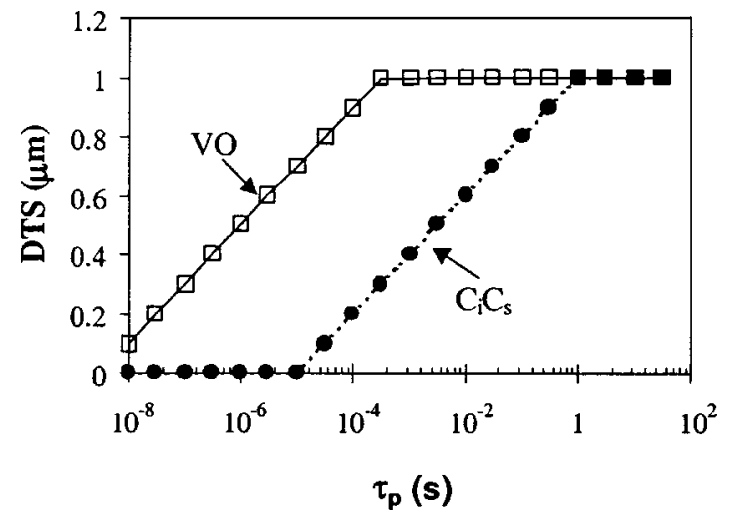

FIG. 7. Shift of the peak position $\left(d_{\max }\right)$ caused by filling in the Debye tail (DTS) for VO (open squares) and $\mathrm{C}_{i} \mathrm{C}_{s}$ (black circles) as a function of the filling pulse duration $\left(\tau_{p}\right)$, as deduced from Fig. 5 .

of transfer from the configuration $\mathrm{B}^{-}$to $\mathrm{A}^{-}$. Therefore, to assume a similar slope of DTS $\left(\tau_{p}\right)$ for $\mathrm{VO}$ and $\mathrm{C}_{i} \mathrm{C}_{s}$ may have a limited validity and is to be regarded as a first approximation. Under these assumptions, it is possible to calculate the $E_{c}-0.17 \mathrm{eV}$ distribution for different values of $\tau_{p}$ and to extract the position of the peak maximum. The values of $d_{\max }$ for the calculated $E_{c}-0.17 \mathrm{eV}$ distributions are given in Fig. 8 with $\Delta$ ranging from 0 to $0.8 \mu \mathrm{m}$ in steps of $0.2 \mu \mathrm{m}$. As in the experimental case, the value of $d_{\max }$ for $\tau_{p}=10^{-6} \mathrm{~s}$ is defined as zero. As revealed by Fig. 8, for $\Delta$ $\leqslant 0.2 \mu \mathrm{m}, d_{\max }$ exhibits a dip around $\tau_{p} \sim 10^{-4}$ s, i.e., when the $\mathrm{C}_{i} \mathrm{C}_{s}$ contribution rises. When $\Delta=0.4 \mu \mathrm{m}$, the dip is changed into a plateau, which disappears for $\Delta \geqslant 0.6 \mu \mathrm{m}$. Hence, the shape of the curve $d_{\max }$ vs $\tau_{p}$ gives by itself some information about $\Delta$. Moreover, the maximum value of $d_{\max }$ increases with $\Delta$. A comparison between the calculated and measured values of $d_{\max }$ for the boron-implanted samples is made in Fig. 9, and within the experimental accuracy, a reasonable agreement is obtained when the calculations are performed with $\Delta$ equal to 0.4 and $0.8 \mu \mathrm{m}$ for the $\alpha=7^{\circ}$ and $\alpha=40^{\circ}$ implants, respectively. The absolute numerical values of $\Delta$ assumed in the calculations should, however, be taken with some care since the value used for the $\alpha=40^{\circ}$

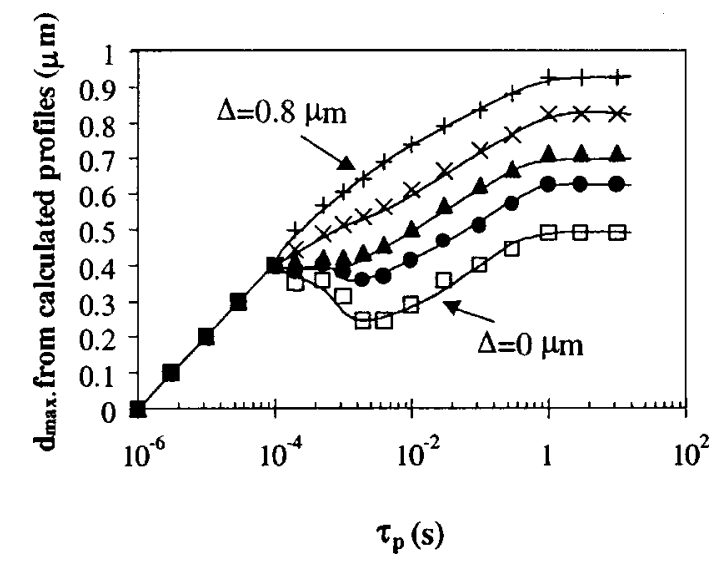

FIG. 8. Position $\left(d_{\max }\right)$ of the maximum concentration of the $E_{c}-0.17 \mathrm{eV}$ depth profile as a function of the filling pulse duration $\left(\tau_{p}\right)$ according to calculations assuming different $\Delta$ values. $\Delta$ ranges from 0 (open squares) to $0.8 \mu \mathrm{m}$ (crosses) in steps of $0.2 \mu \mathrm{m}$. 


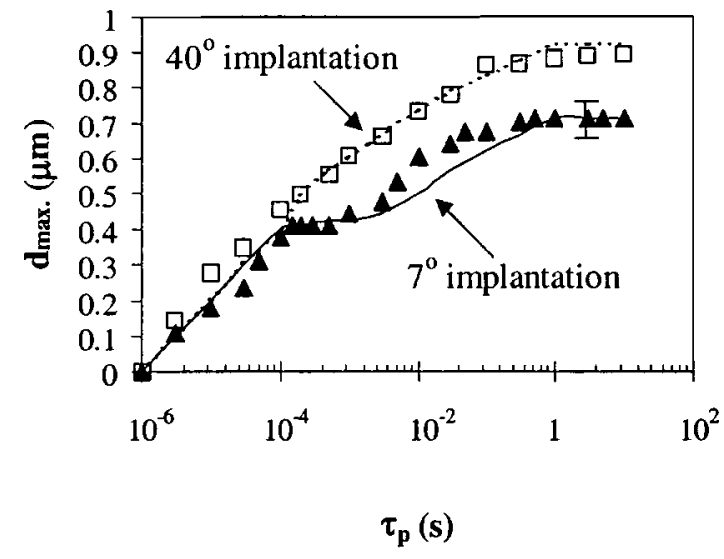

FIG. 9. Comparison between experimental (symbols) and calculated (lines) values of $d_{\max }$ as a function of the filling pulse duration $\left(\tau_{p}\right)$ for two boronimplanted samples. One sample is implanted with $1.4 \times 10^{8} / \mathrm{cm}^{2}$ B $(6.8$ $\mathrm{MeV}$ ) ions at $\alpha=7^{\circ}$ (black triangles and continuous line) while the other one is implanted with $1 \times 10^{8} / \mathrm{cm}^{2} \mathrm{~B}(6.8 \mathrm{MeV})$ ions at $\alpha=40^{\circ}$ (open squares and dotted line). In the calculations $\Delta$ is put equal to 0.4 and $0.8 \mu \mathrm{m}$ for the $\alpha=7^{\circ}$ and $\alpha=40^{\circ}$ implants, respectively.

implantation $(0.8 \mu \mathrm{m})$ is higher than the measured one $(\Delta$ $\sim 0.6 \mu \mathrm{m})$. Further, the experimental and calculated values for $d_{\max }$ deviate somewhat in the $\left(10^{-3}-10^{-1}\right) \mathrm{s}$ range when $\alpha=7^{\circ}$. Both these observations indicate that the slope of DTS vs $\tau_{p}$ should be larger for $\mathrm{C}_{i} \mathrm{C}_{s}$ than for VO. However, the general trend of the experimental data is confirmed by the calculations, and especially, the increase of $\Delta$ with $\alpha$ is reproduced. A physical model consistent with this observation as well as with the dependence of $\Delta$ on ion energy is discussed in the following section.

\section{E. Separation of vacancy and interstitial depth profiles: Physical model}

In contrast to that for the forward momentum, it is interesting to note that the width of the vacancy (and interstitial) distributions, as calculated by TRIM, increases in the direction parallel to the surface normal as $\alpha$ increases. Following this observation, the $\Delta$ values displayed in Table II are plotted in Fig. 10 as a function of the full width at half maximum (FWHM) for the vacancy depth profile as obtained from TRIM 98. A close correlation is found and it can be associ-

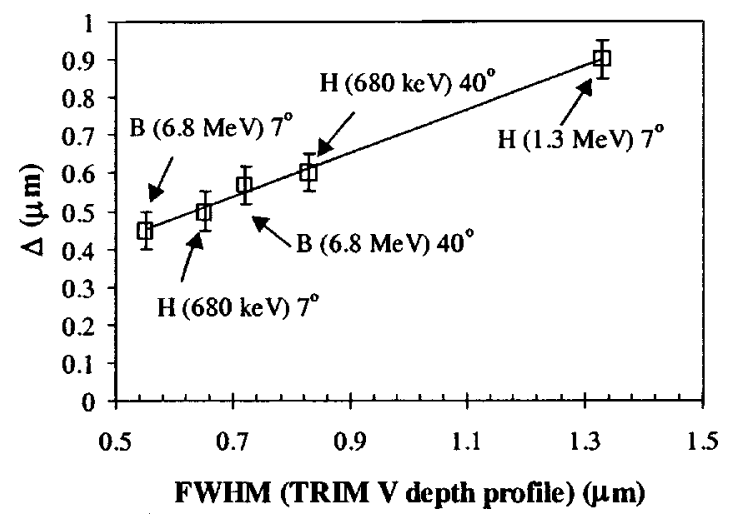

FIG. 10. Experimental values of $\Delta$ vs the full width at half maximum (FWHM) of the vacancy depth profile as calculated by TRIM 98 .
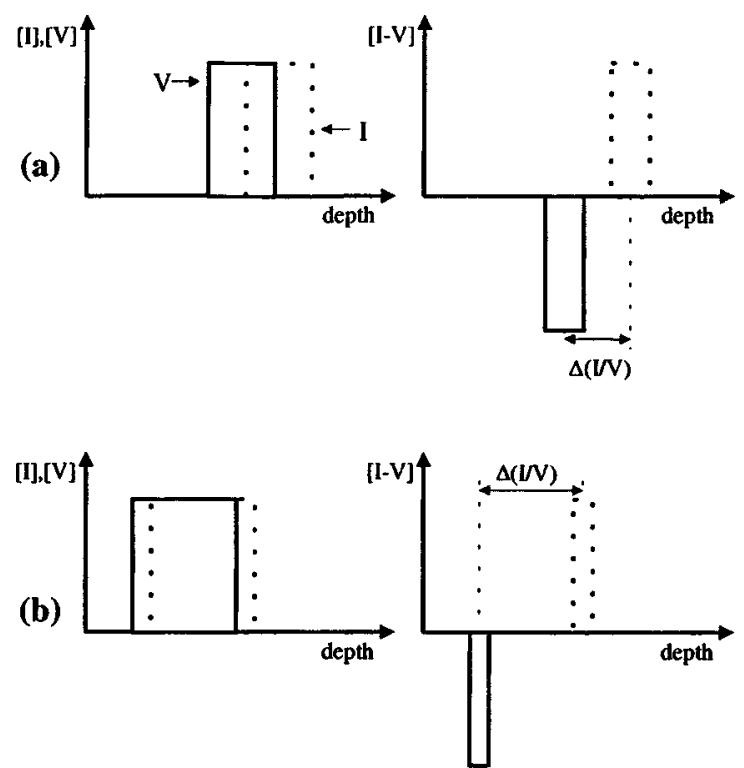

FIG. 11. Schematics of two implantation conditions with $\alpha$ equal to $7^{\circ}$ (a) and $40^{\circ}$ (b). Initial $I$ (dotted line) and $V$ (continuous line) distributions before/after direct annihilation are on the left/right-hand side of the figure. Direct annihilation is assumed to be perfect in the overlapping regions.

ated with the interpretation illustrated schematically in Fig. 11. We assume for simplicity that the distributions of point defects are box like in shape and that the direct annihilation of overlapping interstitials and vacancies is perfect. Figure 11(a) represents the $\alpha=7^{\circ}$ implantation before (on the left) and after (on the right) annihilation while Fig. 11(b) represents the $\alpha=40^{\circ}$ implantation. The forward momentum relative to the surface normal is less in the second case, and accordingly, the separation between the initial $I$ and $V$ distributions is smaller. On the other hand, the $I$ and $V$ distributions are wider for $\alpha=40^{\circ}$, and as a result, the separation between the $I$ and $V$ distributions after annihilation $[\Delta(I / V)]$ is larger in this case. Thus, $\Delta$ increases as $\alpha$ increases or, more generally, as the FWHM of the $V$ (or $I$ ) distribution increases. This is in accordance with Fig. 10 where also the energy dependence of $\Delta$ for protons at a fixed angle of incidence $\left(\alpha=7^{\circ}\right)$ is shown to obey this correlation. It should be emphasized that the initial shift between the $I$ and $V$ distributions due to the preferential forward momentum is of the order of nanometers while the final shift after annihilation $[\Delta(I / V)]$ is in the micrometer range. Therefore, Fig. 11 is only a schematic representation.

The basic assumption of perfect direct annihilation of the overlapping one-dimensional distributions of $I$ and $V$ in Fig. 11 ignores separation of the point defects in the lateral directions, perpendicular to the incoming ion beam. Further, the width and absolute values of the experimental profiles are substantially larger than those of the distributions deduced by taking the difference between the one-dimensional TRIM profiles of the $I$ and $V$. However, the integrated density of VO centers in, for example, Fig. 4 amounts only to $\sim 1 \%$ of the initial vacancy density generated by the implantation, according to TRIM calculations. Hence, although not perfect, the direct annihilation is very efficient and the oversimplified model schematically displayed in Fig. 11 appears to be suf- 
ficiently valid to account for the main mechanisms underlying the observed shift between the interstitial and vacancy distributions.

\section{CONCLUDING REMARKS}

We have studied the shift between the depth distributions of implantation-induced vacancies and self-interstitials and for all the implantation conditions investigated, the interstitial $(I)$ depth distribution is deeper than the vacancy $(V)$ one. This holds even for the "lightest" implantation undertaken: $\mathrm{H}(680 \mathrm{keV})$ with an ion incidence angle of $40^{\circ}$ relative to the surface normal. The experimental concept used is based on the DLTS filling-pulse technique, and thus limited to low ion doses where isolated collision cascades dominate. However, the observed shift $(\Delta)$ between the $I$ and $V$ depth distribution is expected to hold also at higher doses where the resulting damage is due to accumulation of single collision cascades.

It has been shown that $\Delta$ increases as the primary defect distribution width increases. This observation can be explained in a simple way: for all the implantation conditions investigated, there is enough preferential forward energy transfer to the recoiling silicon atoms to create a small shift between the initial $I$ and $V$ distributions. The direct recombination (or $I / V$ annihilation) is so efficient that the final shift $\Delta$ after annihilation increases as the width of the overlap of the $I$ and $V$ distributions increases, i.e., as the primary defect distribution width increases. A close correlation between $\Delta$ and the primary defect distribution width is demonstrated by varying the angle of ion incidence as well as the ion energy.

Finally, it may be pointed out that the direct annihilation of $I$ and $V$ is efficient but not perfect. For instance, each boron ion with an energy of $6.8 \mathrm{MeV}$ gives rise to $\sim 10 \mathrm{VO}$ centers. Nevertheless, in the so-called " +1 model," ${ }^{26}$ direct annihilation is assumed to be perfect and only one interstitial per ion is considered to remain after implantation. The +1 model is used as a starting point in some models dealing with extended defect formation ( $\{311\}$ defects, ${ }^{27}$ dislocations, ${ }^{28,29}$ boron clusters $^{30}$ ) and/or the transient-enhanced diffusion phenomenon. ${ }^{26}$ It is obviously a questionable starting point and the use of a more-refined model including a shift between the initial $I$ and $V$ distributions may be helpful for further understanding and improved modeling.

\section{ACKNOWLEDGMENTS}

The authors gratefully acknowledge support from the European Commission TMR Program, network Contract No.
ERBFMRXCT 980228. Partial financial support was also received from the Swedish Research Council for Engineering Science (TFR) and the Swedish Foundation for International Cooperation in Research and Higher Education (STINT).

${ }^{1}$ T. Alväger and N. Hansen, Rev. Sci. Instrum. 33, 567 (1962).

${ }^{2}$ P. A. Stolk, H.-J. Gossmann, D. J. Eaglesham, D. C. Jacobson, C. S. Rafferty, G. H. Gilmer, M. Jaraiz, J. M. Poate, H. S. Luftman, and T. E. Haynes, J. Appl. Phys. 81, 6031 (1997).

${ }^{3}$ P. M. Fahey, P. B. Griffin, and J. D. Plummer, Rev. Mod. Phys. 61, 289 (1989).

${ }^{4}$ V. Raineri, R. J. Schreutelkamp, F. W. Saris, K. T. F. Jansen, and R. E. Kaim, Appl. Phys. Lett. 58, 922 (1991).

${ }^{5}$ K. Kyllesbech Larsen, V. Privitera, S. Coffa, F. Priolo, C. Spinella, M. Saggio, and S. U. Campisano, Nucl. Instrum. Methods Phys. Res. B 112, 139 (1996).

${ }^{6}$ E. G. Roth, O. W. Holland, V. C. Venezia, and B. Nielsen, J. Electron. Mater. 26, 1349 (1997).

${ }^{7}$ V. C. Venezia, T. E. Haynes, A. Agarwai, L. Pelaz, H.-J. Gossmann, D. C. Jacobson, and D. J. Eaglesham, Appl. Phys. Lett. 74, 1299 (1999).

${ }^{8}$ L. Pelaz, G. H. Gilmer, M. Jaraiz, S. B. Herner, H.-J. Gossmann, D. J. Eaglesham, G. Hobler, C. S. Rafferty, and J. Barbolla, Appl. Phys. Lett. 73, 1421 (1999).

${ }^{9}$ S. Coffa, V. Privitera, F. Priolo, S. Libertino, and G. Mannino, J. Appl. Phys. 81, 1639 (1997).

${ }^{10}$ P. Pellegrino, P. Lévêque, J. Wong-Leung, C. Jagadish, and B. G. Svensson, Appl. Phys. Lett. 78, 3442 (2001).

${ }^{11}$ D. V. Lang, J. Appl. Phys. 45, 3023 (1974).

${ }^{12}$ J. P. Biersack and L. G. Haggmark, Nucl. Instrum. Methods 174, 257 (1980); J. F. Ziegler, J. P. Biersack, and U. Littmark, in The Stopping and Range of Ions in Solids, edited by J. F. Ziegler (Pergamon, New York, 1985), Vol. 1.

${ }^{13}$ A. Hallén, P. A. Ingemarsson, P. Håkansson, G. Possnert, and B. U. R. Sundqvist, Nucl. Instrum. Methods Phys. Res. B 36, 345 (1989).

${ }^{14}$ B. G. Svensson, K.-H. Rydén, and B. M. S. Lewerentz, J. Appl. Phys. 66, 1699 (1989).

${ }^{15}$ L. C. Kimerling, Inst. Phys. Conf. Ser. 31, 221 (1977).

${ }^{16}$ B. G. Svensson and M. Willander, J. Appl. Phys. 62, 2758 (1987)

${ }^{17}$ A. O. Evwaraye and E. Sun, J. Appl. Phys. 47, 3776 (1976).

${ }^{18}$ G. D. Watkins and J. W. Corbett, Phys. Rev. 121, 1001 (1961); J. W. Corbett, G. D. Watkins, R. M. Chrenko, and R. S. McDonald, ibid. 121, 1015 (1961).

${ }^{19}$ L. W. Song, X. D. Zhan, B. W. Benson, and G. D. Watkins, Phys. Rev. B 42, 5765 (1990).

${ }^{20}$ G. E. Jellison, Jr., J. Appl. Phys. 53, 5715 (1982).

${ }^{21}$ J. Frenkel, Phys. Rev. 54, 647 (1938).

${ }^{22}$ L. C. Kimerling and J. L. Benton, Appl. Phys. Lett. 39, 410 (1981).

${ }^{23}$ A. K. Tripping and R. C. Newman, Semicond. Sci. Technol. 2, 315 (1987).

${ }^{24}$ A. Zylberztejn, Appl. Phys. Lett. 33, 200 (1978).

${ }^{25}$ D. Pons, J. Appl. Phys. 55, 3644 (1984).

${ }^{26}$ M. D. Giles, J. Electrochem. Soc. 138, 1160 (1991).

${ }^{27}$ D. J. Eaglesham, P. A. Stolk, H.-J. Gossmann, and J. M. Poate, Appl. Phys. Lett. 65, 2305 (1994).

${ }^{28}$ K. S. Jones and D. Venables, J. Appl. Phys. 69, 2931 (1991).

${ }^{29}$ J. K. Listebarger, K. S. Jones, and J. A. Slinkman, J. Appl. Phys. 73, 4815 (1993).

${ }^{30}$ M. B. Huang and I. V. Mitchell, J. Appl. Phys. 85, 174 (1999). 